\title{
Update on Targeted Therapy in Medullary Thyroid Cancer
}

\author{
Christian Okafor ${ }^{1}$, Julie Hogan ${ }^{1}$, Margarita Raygada ${ }^{1}$, Barbara J. Thomas ${ }^{1}$, \\ Srivandana Akshintala ${ }^{1}$, John W. Glod ${ }^{1}$ and Jaydira Del Rivero ${ }^{2 *}$ \\ 1 Pediatric Oncology Branch, Center for Cancer Research, National Cancer Institute, National Institutes of Health, Bethesda, \\ MD, United States, ${ }^{2}$ Developmental Therapeutics Branch, Center for Cancer Research, National Cancer Institute, National \\ Institutes of Health, Bethesda, MD, United States
}

Medullary thyroid carcinoma (MTC) is a rare neuroendocrine tumor that accounts for $2-4 \%$ of all thyroid cancers. All inherited MTC and approximately 50\% of sporadic cases are driven by mutations in the REarranged during Transfection (RET) proto-oncogene. The recent expansion of the armamentarium of RET-targeting tyrosine kinase inhibitors (TKIs) has provided effective options for systemic therapy for patients with metastatic and progressive disease. However, patients that develop resistant disease as well as those with other molecular drivers such as RAS have limited options. An improved understanding of mechanisms of resistance to TKls as well as identification of novel therapeutic targets is needed to improve outcomes for patients with MTC.

Keywords: medullary thyroid cancer (MTC), MEN2, calcitonin, tyrosine kinase inhibitor, clinical trials

\section{BACKGROUND}

Medullary thyroid carcinoma (MTC) is a rare neuroendocrine tumor that arises from the calcitoninsecreting parafollicular cells (C-cells). It has an estimated annual incidence ranging from 0.14 to $0.21 /$ 100,000 population and accounts for $2-4 \%$ of all cases of thyroid cancer (1-3). All reported cases of hereditary MTC as well as $40-50 \%$ of sporadic cases are attributed to activating mutations in the REarranged during Transfection (RET) proto-oncogene (3). The RET gene encodes a transmembrane receptor tyrosine kinase that regulates a wide variety of cell processes such as survival, proliferation, motility, and apoptosis. Activating mutations in RAS underly approximately $40 \%$ of non-RET mutated sporadic MTC with most of the remaining cases having no identified oncogenic driver (4, 5). While RET has proved to be an effective therapeutic target, resistant disease can develop in the face of RET-targeting tyrosine kinase inhibitors (TKIs) and options for systemic therapy of non-RET driven MTC are limited. We will review the development of RET-targeting TKIs and ongoing efforts to understand and circumvent mechanisms of resistance. We will also discuss new targets for the systemic therapy of MTC including novel molecular targets, immunotherapy, and the ongoing challenges of developing an effective method of targeting RAS-driven tumors.

\section{INITIATION OF SYSTEMIC THERAPY}

The optimal timing for initiation of TKI therapy is unclear. Currently, systemic therapy should be reserved for patients with clear radiographic progression of disease or those with symptomatic disease. 
There is no evidence that TKI therapy is curative and there have been no completed phase III studies comparing approved agents. It has been recommended that the choice of TKI usage be patientcentered with initiation of therapy depending on patient medical history, concomitant medications, comorbidities and tumor characteristics (6). While RET-targeting TKIs have provided clinical benefit to patients with MTC, the development of resistant disease can occur and the toxicity of both cabozantinib and vandetanib limit their use in patients with small volume, asymptomatic or indolent disease. ATA guidelines recommend considering initiation of TKI therapy for patients with radiographic evidence of disease progression or symptomatic disease (7). The National Comprehensive Cancer Network (NCCN) guidelines (V 3.2020) recommend disease monitoring for patients that are asymptomatic with treatment with TKIs considered for disease that is unresectable or progressing by RECIST criteria (8).

\section{EXPLOITING RET AS A THERAPEUTIC TARGET}

\section{RET Signaling in MTC}

The RET gene encodes a transmembrane receptor tyrosine kinase important in the regulation of cell growth, proliferation, migration, and survival (9). Physiological signaling through the RET receptor occurs upon binding of one of a family of glial cellline derived neurotrophic factor (GDNF) ligands to a glycosylphosphatidylinositol-anchored coreceptor of the RET/ GDNF family receptors alpha family $(G F R \alpha)$. This interaction leads to the formation of a dimeric GDNF-GFR $\alpha$-RET complex and activation of the intracellular tyrosine kinase domains of both RET components of the dimer (10). (Figure 1) Physiological signaling through RET occurs following the phosphorylation of intracellular tyrosine residues on the RET protein that mediate the activation of multiple intracellular signaling pathways including PI3K/AKT, MAPK, JNK and others $(10,11)$. Oncogenic activating mutations of RET have been identified at several sites throughout the protein. Mutations in the cytosine-rich extracellular domain occur at codon C634 of exon 11 as well as 609, 611, 618, or 620 of exon 10. Mutation of these cysteine residues leads to formation of intermolecular disulfide bonds which result in covalent RET dimerization and kinase activation $(12,13)$. Germline mutations at these sites lead to MEN2A. The RET p.M918T mutation associated with MEN2B is in the intracellular tyrosine kinase domain 2, this amino acid change increases ATP binding affinity and leads to constitutive kinase activity in the absence of RET dimerization (12).

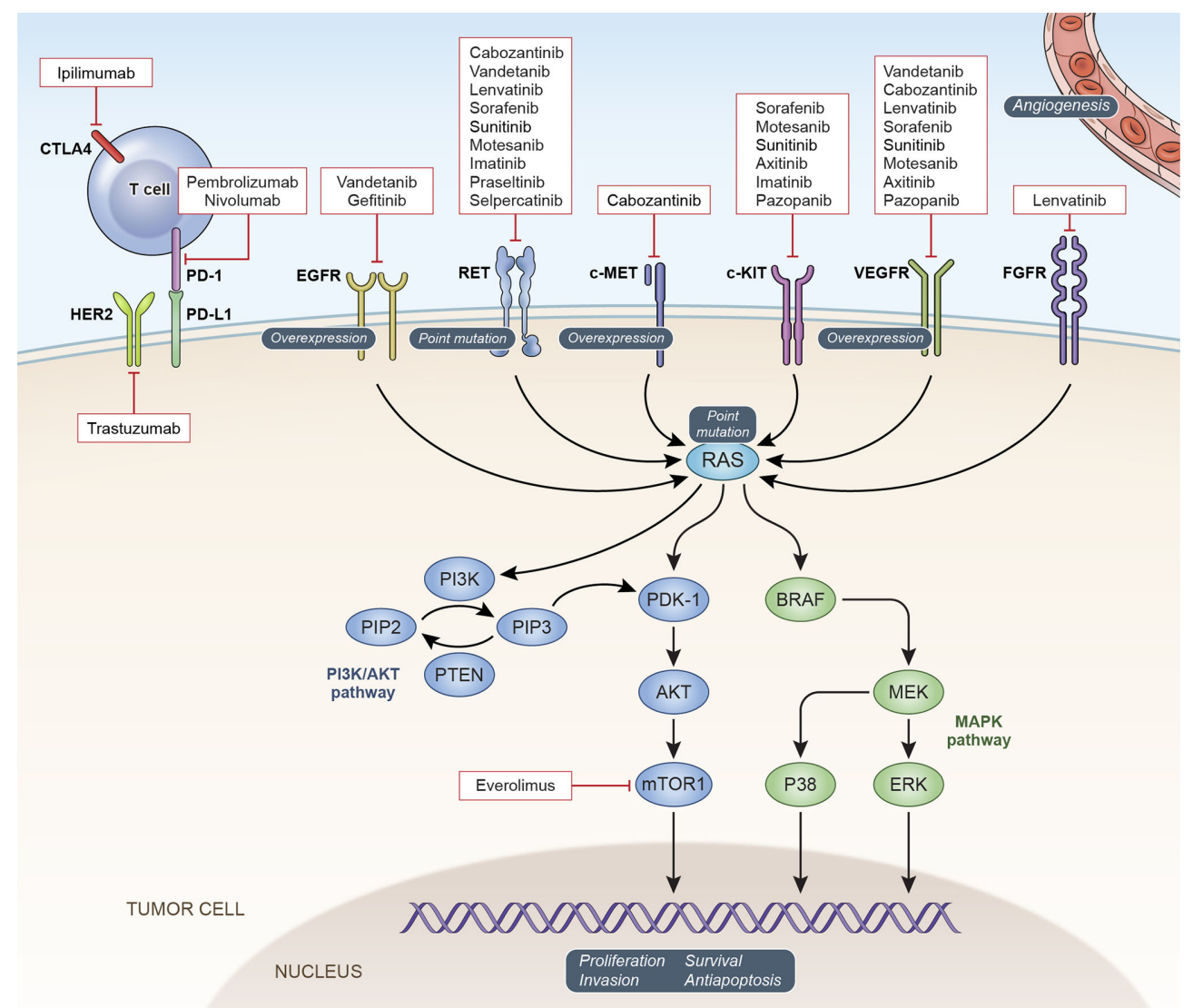

FIGURE 1 | Oncogenic Signaling Pathways in MTC: Potential and confirmed therapeutic targets in MTC are depicted with currently available targeted agents. 


\section{Multi-Target Tyrosine Kinase Inhibitors}

Disruption of RET signaling was first tested as a strategy for the treatment of patients with MTC using the multi-target kinase inhibitor, vandetanib (14). A 20\% partial response rate was seen in patients treated on this phase II study. Vandetanib inhibits the activity of RET as well as other receptor tyrosine kinases including VEGFR-2, VEGFR-3, and EGFR (15) and the inhibition of each of these tyrosine kinases may play a role in the impact of vandetanib on MTC tumor growth. The drug was approved by the Food and Drug Administration (FDA) in April 2011 for the treatment of patients with symptomatic or progressive MTC with unresectable locally advanced or metastatic disease based on the results of a phase III trial which demonstrated a statistically significant improvement in 6-month progression-free survival (PFS) of $83 \%$ for patients treated with vandetanib compared to $63 \%$ for placebo (16). Importantly, the approval did not require the presence of a RET activating mutation. In 298 patients with sporadic MTC enrolled in this study a RET mutation was documented in 155 patients with no RET mutation in 8 patients and RET mutation status unknown in 135 patients. Cabozantinib, another multitarget TKI approved by the FDA for the treatment of patients with progressive or symptomatic MTC demonstrated an improved median PFS of 11.2 months compared to 4 months in a placebo group (17). A long-term follow-up analysis of this study after a minimum of 42 months did not show a statistically significant increase in overall survival with cabozantinib compared to placebo, although a non-significant 5.5-month increase was reported (18). A follow-up analysis showed an overall survival of 44.3 months in patients with tumors with RET M918T mutations treated with cabozantinib compared to 18.9 months for those treated with placebo. The difference in OS between cabozantinib and placebo treated patients who did not have M918T mutated tumors was 20.2 and 21.5 months (18). Because the cabozantinib study enrolled only patients with radiographic evidence of progressive disease compared to the initial phase 3 study evaluating vandetanib, which did not required evidence of disease progression for enrollment, it is difficult to compare the efficacy of the two agents. In addition to these studies in adult patients both vandetanib and cabozantinib were also effective for the treatment of pediatric patients with MTC (19-21). Despite these successes neither vandetanib nor cabozantinib leads to complete responses in patients with MTC and the development of resistant disease is a significant problem. As a consequence of their broad kinase inhibitor activity both of these agents have a number of toxicities. Toxicities seen with vandetanib included diarrhea, rash, nausea, hypertension, and headache (16) and patients who received cabozantinib, which inhibits hepatocyte growth factor receptor (MET) and VEGFR2 in addition to RET often experienced diarrhea, palmar-plantar erythrodysesthesia, decreased weight and appetite, nausea, and fatigue (17). The modest activity as well as the significant sideeffects of multi-target TKIs can limit their utility in the treatment of patients with MTC.

Other multi-target TKIs have been investigated for the treatment of patients with MTC (Table 1). Sunitinib had an objective response rate of $38.5 \%$ with median PFS of 16.5 and OS of 29.4 months in a group of 26 patients (30). In a study of lenvatinib in 59 patients with MTC an objective response rate of $36 \%$ was reported (31). The trial included patients with both RET-driven and non-RET-driven disease and RET mutation status was not associated with response or PFS. Responses and clinical benefit have also been reported in patients with MTC with other multi-target TKIs including sorafenib $(22,32)$, and Anlotinib (23). Taken together, responses in patients with both RET and RAS-driven disease to TKIs with a range of targets suggests that the targeting of multiple signaling pathways could provide therapeutic opportunities for patients with advanced MTC. Additional TKIs including the multi-target agent regorafenib as well as next-generation RET-targeting TKIs are currently being evaluated (Table 2 ).

\section{RET-Specific Tyrosine Kinase Inhibitors}

The recently approved TKIs, selpercatinib and pralsetinib have more specific RET-targeting activity which leads to an improved side effect profile. In a phase 1-2 study of selpercatinib in patients with progressive $R E T$-mutant MTC a $69 \%$ response rate was seen in patients who had previously received vandetanib or

TABLE 1 | Summary of results of completed tyrosine kinase inhibitor trials.

\begin{tabular}{|c|c|c|c|c|c|c|}
\hline Tyrosine Kinase Inhibitors & Target(s) & Mutations & ORR & mPFS (months) & mOS (months) & Clinical Trial ID \\
\hline Vandetanib (22) & VEGFR2-3, EGFR, RET & RET+RAS+UK & $45 \%$ & 30.5 & NR & NCT00410761 \\
\hline \multirow{3}{*}{ Cabozantinib (23) } & VEGFR2, KIT, FLT-3, RET, MET & RET+RAS+UK & $28 \%$ & 11.2 & 26.6 & NCT00704730 \\
\hline & & M918T Negative & $20 \%$ & 20.2 & 5.7 & \\
\hline & & M918T & $34 \%$ & 13.9 & 44.3 & \\
\hline \multirow[t]{2}{*}{ Selpercatinib (24) } & RET, VEGFR2 & RET/Previous TKI & $69 \%$ & NR (1-year PFS 82\%) & NR & NCT03157128 \\
\hline & & RET/TKI Naïve & $73 \%$ & NR (1-year PFS 92\%) & NR & \\
\hline Sorafenib (25) & BRAF, KIT, FLT-3, VEGFR2, PDGFR & ND & $25 \%$ & NR & NR & NCT02114658 \\
\hline Lenvatinib (26) & VEGFR1-3, FGFR1-4, PDGFR $\alpha$, KIT, RET & RET+RAS & $36 \%$ & 9 & 16.6 & NCT00784303 \\
\hline Anlotinib (27) & VEGFR1-3, FGFR1-4, KIT FGFR & ND & $48.4 \%$ & 22.4 & 50.4 & NCT02586350 \\
\hline \multirow[t]{2}{*}{ Pralsetinib (28) } & RET, VEGFR2 & RET/Previous TKI & $60 \%$ & NR & NR & NCT03037385 \\
\hline & & RET/TKI Naïve & $71 \%$ & NR & NR & \\
\hline Sunitinib (29) & PDGFR, KIT VEGFR1-3, FLT-3, RET & ND & $38.5 \%$ & 16.5 & 29.4 & NCT00510640 \\
\hline
\end{tabular}

Completed trials with corresponding references are included. Mutation status is indicated as RET altered (RET), RAS altered (RAS), unknown (UK), and not determine (ND). Objective response rate (ORR), Progression free survival (PFS), and overall survival (OS) are indicated. NR indicates that median PFS or OS have not been reached. 
TABLE 2 | Ongoing early phase trials for patients with MTC.

\begin{tabular}{|c|c|c|c|c|}
\hline Drug & Molecular Target & Phase & Eligibility & Clinical Trial ID \\
\hline Regorafenib & BRAF, VAGFR1-3 PDGFR $\alpha / \beta$, RET, KIT, FGFR1-2 & 2 & MTC & NCT02657551 \\
\hline TPX-0046 & RET & $1 / 2$ & RET altered tumors & NCT04161391 \\
\hline TAS0953/HM06 & RET & $1 / 2$ & RET altered tumors & NCT04683250 \\
\hline GFR $\alpha 4$ CAR T Cells & GFR $\alpha 4$ & 1 & MTC & NCT04877613 \\
\hline
\end{tabular}

Currently open early phase studies including the multi-target TKI regorafenib as well as next generation RET-targeting TKIs and the first CAR T-cell targeting MTC.

cabozantinib with a $73 \%$ response rate in TKI-naïve patients (33). The drug was well tolerated with grade 3 adverse events including hypertension (21\%) and diarrhea (6\%). Results of a study of pralsetinib in patients with MTC were recently reported with a response rate of $71 \%$ in TKI-naïve patients and $60 \%$ in patients who had previously received vandetanib or cabozantinib and pralsetinib was also FDA-approved for the treatment of patients with RET altered thyroid cancer (34). Selpercatinib was also effective and well-tolerated in five pediatric patients with cancers with activating mutations in RET including two patients with MTC (35). Selpercatinib and pralsetinib have several potential advantages compared to the multi-targeted TKIs including an improved side effect profile as well as activity against RET V804L and V804M mutants. However, resistance has also emerged to these newer TKIs. Solvent front mutations at RET glycine 810 as well as mutations at RET Y806 within the hinge region of the kinase cause resistance to both selpercatinib and pralsetinib $(24,36)$. Amplification of the MET gene has also been identified as a mechanism of resistance to selpercatinib in RET fusion non-small cell lung cancer (28). A combination of selpercatinib and the MET inhibitor crizotinib was able to overcome resistance due to MET amplification (28).

\section{TARGETS DOWNSTREAM OF RET}

The success of RET inhibitors in the treatment of MTC suggests that downstream signaling pathways activated by RET may also be effective therapeutic targets. While the mechanisms that induce MTC oncogenesis through RET activation have not been completely defined, there is data supporting the involvement of a number of cellular signaling pathways in this process (Figure 1). The phosphatidylinositol 3-kinase (PI3K)/ $\mathrm{AKT} / \mathrm{mammalian}$ target of rapamycin (mTOR) pathway is activated in preclinical MTC models as well as cases of MTC $(37,38)$. This data as well as the efficacy of everolimus in other neuroendocrine tumors such as pancreatic NET led to its evaluation in patients with MTC. Responses have been reported in patients with MTC in several trials of the mTOR inhibitor everolimus $(29,39,40)$. While dramatic response rates were not observed, this work provides additional evidence that the $\mathrm{PI} 3 \mathrm{~K} / \mathrm{AKT} / \mathrm{mTOR}$ pathway is important in MTC and may provide additional therapeutic targets for a subgroup of patients. Combined inhibition of both the RAS/MEK/ERK and PI3K/ $\mathrm{AKT} / \mathrm{mTOR}$ pathways has demonstrated activity in preclinical studies of MTC $(25,26)$. Unfortunately, combined inhibition of both pathways has thus far been limited by toxicity in early clinical trials $(27,41)$.

\section{RAS-DRIVEN MTC}

\section{RAS Mutant MTC}

Approximately 70\% of cases of MTC without an identifiable RET mutation have been attributed to RAS gene mutations (42-45). $R A S$ genes code for a family of cellular signaling GTPases that regulate a wide range of downstream pathways including MEK/ ERK and PI3K/AKT and play a role in numerous functions including cell growth and proliferation, apoptosis, and differentiation (45-47). Approximately $20 \%$ of all cancers have activating mutations in RAS (48). Activating mutations in HRAS are the most commonly seen in MTC with a lesser percentage of KRAS and rare NRAS mutations $(44,49,50)$. RAS and RET mutations are typically mutually exclusive in MTC except for a small number of anecdotal reports with mutations in both genes (45). As with other RAS-driven cancers, the mutations seen in MTC occur primarily in exons 2 and $3(45,49)$. The most common activating mutations in RAS occur at G12, G13, and Q61 (51).

\section{Strategies for Targeting RAS}

Targeting RAS and its associated signaling pathways has proven to be challenging. RAS has a lack of sites amenable to small molecule inhibitor binding and is difficult to target pharmacologically (52). RAS pathway inhibitors have been investigated for the treatment of MTC. In a phase I trial of the multi-kinase inhibitor sorafenib in combination with the farnesyltransferase inhibitor tipifarnib 5/13 patients with MTC showed a response, however the mutation status of the patients for RET and RAS was not reported (53). Post-hoc analysis was performed on data from a phase III trial of cabozantinib for MTC. Patients with cancers with mutations in RAS (HRAS, KRAS, and $N R A S$ ) had an overall response rate of $31 \%$ compared to a response rate of $32 \%$ in patients with $R E T$-mutation positive disease with no survival benefit observed in patients with tumors lacking both RET and RAS mutations (54). Unlike KRAS and NRAS, HRAS is prenylated only by farnesyltransferase (55). The farnesyltransferase inhibitor tipifarnib is being tested in patients with HRAS driven cancers and patients with HRAS mutated MTC are eligible (NCT02383927).

Several novel strategies are currently being explored for the treatment of patients with RAS mutant cancers. A number of small molecule inhibitors of KRAS-G12C have recently been 
developed and are being clinically evaluated (56-58). Sotorasib and adagrasib are in phase II trials. Sotorasib was investigated in patients with cancers with KRAS-G12C mutation and had a disease response in approximately $30 \%$ of patients with nonsmall cell lung cancer (59). Other targeted inhibitors of specific mutated activated RAS isoforms are in clinical development (58). Activating mutations of RAS have also been targeted using immunological approaches. Infusion of expanded autologous KRAS-G12D-specific CD-8+ tumor infiltrating lymphocytes led to a response in a patient with RAS mutant colorectal carcinoma (60). Additional work has identified T-cell receptors that target activating mutations in KRAS (61-63). Based on this and other work early-stage trials are now underway using KRAS G12V and G12D-specific TCR cell therapy approaches (NCT04146298; NCT03190941; NCT03745326). While these approaches hold considerable promise, they are limited by specificity for a specific RAS isoform and mutation as well as the HLA restriction of TCR-based treatments.

\section{IMMUNOTHERAPY AND OTHER TARGETED THERAPIES}

\section{Peptide Receptor Radionuclide Therapy}

Somatostatin receptor is present on a majority of cases of MTC (64) and can be detected with somatostatin receptorbased PET modalities (65). While no randomized controlled trials of Peptide Receptor Radionuclide Therapy (PRRT) in MTC have been done to date the use of radiolabeled somatostatin analogs for the treatment of MTC has been reported in a number of smaller studies. Patients treated with 90Y-DOTATOC had a response rate of 29\% (9 of 31 patients) in a phase II study (66). In a single-institution retrospective analysis of patients with MTC treated with ${ }^{177} \mathrm{Lu}$ DOTATATE, a $62 \%$ response rate (27 of 43 patients) based on RECIST 1.1 criteria was reported (67). Recent reviews have summarized the published experience of PRRT in the treatment of patients with medullary thyroid cancer. Overall cumulative objective response rates to PRRT in patients with MTC of 5.1 and $10.6 \%$ were reported $(68,69)$. While these studies do show promise, the lack of controlled studies of PRRT in MTC as well as the potential for significant sideeffects including hematologic and nephrotoxicity point to the need for randomized controlled trials in this area.

\section{Other Immunotherapeutic Approaches}

Immunotherapy approaches are being tested for the treatment of patients with MTC including specific antigen-targeting approaches as well as strategies that leverage the anti-tumor immune response. Tumor vaccines incorporating the MTC secretory product carcinoembryonic antigen (CEA) have been studied in early phase clinical trials. An early study using vaccines generated with autologous mature dendritic cells loaded with calcitonin and CEA were studied in a small study of 7 patients. A decrease in calcitonin and CEA in 3/7 patients with one patient showing complete regression in metastatic nodules was reported in this trial (70). The CEA vaccine, GI6207, was investigated in a phase 1 trial that enrolled 25 patients with metastatic CEA-expressing carcinomas and included one patient with MTC (71). The single patient with MTC on this study did not have a radiographic response, however, a marked inflammatory reaction at sites of metastatic disease was observed as well as significant $\mathrm{T}$ cell responses. Immune checkpoint inhibitors are also being explored in the treatment of MTC. A patient with recurrent MTC who was treated with a yeast-CEA cancer vaccine followed by anti-PD-L1 inhibitor, avelumab, was reported to have a $40 \%$ decrease in his calcitonin levels with radiographic stable disease. The tumor was found to express PDL1 which may explain the calcitonin response to immunotherapy (72). Adoptive T-cell therapy has been successful in the treatment of patients with acute lymphoblastic leukemia and the strategy is being used to target other cancer types. Bhoj and colleagues reported preclinical data demonstrating that chimeric antigen receptor T-cells targeting GDNF family receptor alpha 4 could eliminate MTC in a murine xenograft model (73). An anti-GDNF $\alpha 4$ CAR T-Cell approach will be tested in a planned phase I trial (NCT04877613) (Table 2). An improved understanding of the immune microenvironment of MTC as well as identification of novel target antigens is needed to develop new effective immunotherapies for patients with MTC.

\section{CONCLUSION}

The development of RET-targeting TKIs has made a substantial impact on the treatment of patients with MTC. Despite this advance, important challenges remain. Patients with sporadic MTC driven by RAS mutations as well as patients with disease resistant to the available RET-targeting TKIs have limited therapeutic options. Important questions remain in optimizing the use of TKIs in the treatment of patients with MTC. As use of the RET-specific TKIs increases it will be important to understand the patterns of resistance to these drugs and nextgeneration RET-targeting tyrosine kinase inhibitors that circumvent new resistance mutations will be needed. Improved understanding of downstream pathways involved in resistance, such as MET signaling, may inform the design of combination trials. Other novel strategies such as intermittent or alternating dosing of RETinhibitors could also be considered. Another major challenge is the optimal timing for the initiation of TKI therapy. Large, randomized trials would be required to determine if initiation of a RET inhibitor prior to radiographic disease progression would provide any clinical benefit. RET-agnostic immunotherapy and antigen-targeting approaches also hold promise for expanding treatment options for MTC. While responses to PRRT have been reported, wellcontrolled prospective trials are still needed to define its role in the treatment of patients with MTC. T-cell based therapies have been highly effective in hematopoietic malignancies and the initial steps in their evaluation and development for the treatment of MTC are ongoing. These therapeutic strategies targeting specific antigens distinct from RET, if effective, would be important advances. Studies of these agents may also improve our understanding of the immunology of MTC as well as stimulating the search for 
additional targetable antigens in MTC. Metastatic MTC is currently a treatable but incurable cancer. However, rapid advances in multiple therapeutic strategies holds promise for an eventual cure for this disease.

\section{AUTHOR CONTRIBUTIONS}

CO prepared the initial draft of the manuscript and revised the manuscript. JG and JR critically reviewed and revised the manuscript. JH, MR, BT, and SA reviewed and revised the

\section{REFERENCES}

1. Miranda-Filho A, Lortet-Tieulent J, Bray F, Cao B, Franceschi S, Vaccarella S, et al. Thyroid Cancer Incidence Trends by Histology in 25 Countries: A Population-Based Study. Lancet Diabetes Endocrinol (2021) 9:225-34. doi: 10.1016/S2213-8587(21)00027-9

2. Ceolin L, Duval M, Benini AF, Ferreira CV, Maia AL. Medullary Thyroid Carcinoma Beyond Surgery: Advances, Challenges, and Perspectives. Endocr Relat Cancer (2019) 26:R499-518. doi: 10.1530/ERC-18-0574

3. Romei C, Ciampi R, Elisei R. A Comprehensive Overview of the Role of the RET Proto-Oncogene in Thyroid Carcinoma. Nat Rev Endocrinol (2016) 12:192-202. doi: 10.1038/nrendo.2016.11

4. Araque KA, Gubbi S, Klubo-Gwiezdzinska J. Updates on the Management of Thyroid Cancer. Horm Metab Res (2020) 52:562-77. doi: 10.1055/a-1089-7870

5. Prete A, Borges de Souza P, Censi S, Muzza M, Nucci N, Sponziello M. Update on Fundamental Mechanisms of Thyroid Cancer. Front Endocrinol (Lausanne) (2020) 11:102. doi: 10.3389/fendo.2020.00102

6. Cabanillas ME, Hu MI, Jimenez C. Medullary Thyroid Cancer in the Era of Tyrosine Kinase Inhibitors: To Treat or Not to Treat-and With Which DrugThose are the Questions. J Clin Endocrinol Metab (2014) 99:4390-6. doi: 10.1210/jc.2014-2811

7. Wells SAJr., Asa SL, Dralle H, Elisei R, Evans DB, Gagel RF, et al. Revised American Thyroid Association Guidelines for the Management of Medullary Thyroid Carcinoma. Thyroid (2015) 25:567-610. and XXXC. doi: 10.1089/ thy.2014.0335

8. N.C.C. Network. Thyroid Carcinoma V 3.2020. (2021). Available at: https:// www.nccn.org/professionals/physician_gls/pdf/thyroid.pdf/.

9. Plaza-Menacho I, Burzynski GM, de Groot JW, Eggen BJ, Hofstra RM. Current Concepts in RET-Related Genetics, Signaling and Therapeutics. Trends Genet (2006) 22:627-36. doi: 10.1016/j.tig.2006.09.005

10. Kawai K, Takahashi M. Intracellular RET Signaling Pathways Activated by GDNF. Cell Tissue Res (2020) 382:113-23. doi: 10.1007/s00441-020-03262-1

11. Mulligan LM. RET Revisited: Expanding the Oncogenic Portfolio. Nat Rev Cancer (2014) 14:173-86. doi: 10.1038/nrc3680

12. Plaza-Menacho I. Structure and Function of RET in Multiple Endocrine Neoplasia Type 2. Endocr Relat Cancer (2018) 25:T79-90. doi: 10.1530/ERC17-0354

13. Mulligan LM, Kwok JB, Healey CS, Elsdon MJ, Eng C, Gardner E, et al. GermLine Mutations of the RET Proto-Oncogene in Multiple Endocrine Neoplasia Type 2A. Nature (1993) 363:458-60. doi: 10.1038/363458a0

14. Wells SA Jr, Gosnell JE, Gagel RF, Moley J, Pfister D, Sosa JA, et al. Vandetanib for the Treatment of Patients With Locally Advanced or Metastatic Hereditary Medullary Thyroid Cancer. J Clin Oncol (2010) 28:767-72. doi: 10.1200/JCO.2009.23.6604

15. Gentile C, Martorana A, Lauria A, Bonsignore R. Kinase Inhibitors in Multitargeted Cancer Therapy. Curr Med Chem (2017) 24:1671-86. doi: 10.2174/0929867324666170112112734

16. Wells SAJr., Robinson BG, Gagel RF, Dralle H, Fagin JA, Santoro M, et al. Vandetanib in Patients With Locally Advanced or Metastatic Medullary Thyroid Cancer: A Randomized, Double-Blind Phase III Trial. J Clin Oncol (2012) 30:134-41. doi: 10.1200/JCO.2011.35.5040 manuscript and provided input. All authors contributed to the article and approved the submitted version.

\section{FUNDING}

This work was supported by the NCI My Pediatric and Adult Rare Tumor (MyPART) Network. The MyPART Network is a group of physicians, researchers, patients, their families, and advocates dedicated to finding treatments and improving the lives of people with rare solid tumors.
17. Elisei R, Schlumberger MJ, Muller SP, Schoffski P, Brose MS, Shah MH, et al. Cabozantinib in Progressive Medullary Thyroid Cancer. J Clin Oncol (2013) 31:3639-46. doi: 10.1200/JCO.2012.48.4659

18. Schlumberger M, Elisei R, Muller S, Schoffski P, Brose M, Shah M, et al. Overall Survival Analysis of EXAM, a Phase III Trial of Cabozantinib in Patients With Radiographically Progressive Medullary Thyroid Carcinoma. Ann Oncol (2017) 28:2813-9. doi: 10.1093/annonc/mdx479

19. Fox E, Widemann BC, Chuk MK, Marcus L, Aikin A, Whitcomb PO, et al. Vandetanib in Children and Adolescents With Multiple Endocrine Neoplasia Type 2B Associated Medullary Thyroid Carcinoma. Clin Cancer Res (2013) 19:4239-48. doi: 10.1158/1078-0432.CCR-13-0071

20. Kraft IL, Akshintala S, Zhu Y, Lei H, Derse-Anthony C, Dombi E, et al. Outcomes of Children and Adolescents With Advanced Hereditary Medullary Thyroid Carcinoma Treated With Vandetanib. Clin Cancer Res (2018) 24:753-65. doi: 10.1158/1078-0432.CCR-17-2101

21. Chuk MK, Widemann BC, Minard CG, Liu X, Kim A, Bernhardt MB, et al. A Phase 1 Study of Cabozantinib in Children and Adolescents With Recurrent or Refractory Solid Tumors, Including CNS Tumors: Trial ADVL1211, a Report From the Children's Oncology Group. Pediatr Blood Cancer (2018) 65: e27077. doi: 10.1002/pbc.27077

22. Ito Y, Onoda N, Ito KI, Sugitani I, Takahashi S, Yamaguchi I, et al. Sorafenib in Japanese Patients With Locally Advanced or Metastatic Medullary Thyroid Carcinoma and Anaplastic Thyroid Carcinoma. Thyroid (2017) 27:1142-8. doi: 10.1089/thy.2016.0621

23. Sun Y, Du F, Gao M, Ji Q, Li Z, Zhang Y, et al. Anlotinib for the Treatment of Patients With Locally Advanced or Metastatic Medullary Thyroid Cancer. Thyroid (2018) 28:1455-61. doi: 10.1089/thy.2018.0022

24. Subbiah V, Shen T, Terzyan SS, Liu X, Hu X, Patel KP, et al. Structural Basis of Acquired Resistance to Selpercatinib and Pralsetinib Mediated by nonGatekeeper RET Mutations. Ann Oncol (2021) 32:261-8. doi: 10.1016/ j.annonc.2020.10.599

25. Bertazza L, Barollo S, Radu CM, Cavedon E, Simioni P, Faggian D, et al. Synergistic Antitumour Activity of RAF265 and ZSTK474 on Human TT Medullary Thyroid Cancer Cells. J Cell Mol Med (2015) 19:2244-52. doi: 10.1111/jcmm.12612

26. Jin N, Jiang T, Rosen DM, Nelkin BD, Ball DW. Synergistic Action of a RAF Inhibitor and a Dual PI3K/mTOR Inhibitor in Thyroid Cancer. Clin Cancer Res (2011) 17:6482-9. doi: 10.1158/1078-0432.CCR-11-0933

27. Bardia A, Gounder M, Rodon J, Janku F, Lolkema MP, Stephenson JJ, et al. Phase Ib Study of Combination Therapy With MEK Inhibitor Binimetinib and Phosphatidylinositol 3-Kinase Inhibitor Buparlisib in Patients With Advanced Solid Tumors With RAS/RAF Alterations. Oncologist (2020) 25: e160-9. doi: 10.1634/theoncologist.2019-0297

28. Rosen EY, Johnson ML, Clifford SE, Somwar R, Kherani JF, Son J, et al. Overcoming MET-Dependent Resistance to Selective RET Inhibition in Patients With RET Fusion-Positive Lung Cancer by Combining Selpercatinib With Crizotinib. Clin Cancer Res (2021) 27:34-42. doi: 10.1158/1078-0432.CCR-20-2278

29. Schneider TC, de Wit D, Links TP, van Erp NP, van der Hoeven JJ, Gelderblom $\mathrm{H}$, et al. Beneficial Effects of the mTOR Inhibitor Everolimus in Patients With Advanced Medullary Thyroid Carcinoma: Subgroup Results of a Phase II Trial. Int J Endocrinol (2015) 2015:348124. doi: 10.1155/2015/348124 
30. Ravaud A, de la Fouchardiere C, Caron P, Doussau A, Do Cao C, Asselineau J, et al. A Multicenter Phase II Study of Sunitinib in Patients With Locally Advanced or Metastatic Differentiated, Anaplastic or Medullary Thyroid Carcinomas: Mature Data From the THYSU Study. Eur J Cancer (2017) 76:110-7. doi: 10.1016/j.ejca.2017.01.029

31. Schlumberger M, Jarzab B, Cabanillas ME, Robinson B, Pacini F, Ball DW, et al. A Phase II Trial of the Multitargeted Tyrosine Kinase Inhibitor Lenvatinib (E7080) in Advanced Medullary Thyroid Cancer. Clin Cancer Res (2016) 22:44-53. doi: 10.1158/1078-0432.CCR-15-1127

32. de Castroneves LA, Negrao MV, de Freitas RM, Papadia C, Lima JVJr., Fukushima JT, et al. Sorafenib for the Treatment of Progressive Metastatic Medullary Thyroid Cancer: Efficacy and Safety Analysis. Thyroid (2016) 26:414-9. doi: 10.1089/thy.2015.0334

33. Wirth LJ, Sherman E, Robinson B, Solomon B, Kang H, Lorch J, et al. Efficacy of Selpercatinib in RET-Altered Thyroid Cancers. N Engl J Med (2020) 383:825-35. doi: 10.1056/NEJMoa2005651

34. Subbiah V, Hu MI, Wirth LJ, Schuler M, Mansfield AS, Curigliano G, et al. Pralsetinib for Patients With Advanced or Metastatic RET-Altered Thyroid Cancer (ARROW): A Multi-Cohort, Open-Label, Registrational, Phase 1/2 Study. Lancet Diabetes Endocrinol (2021) 9(8):491-501. doi: 10.1016/s22138587(21)00120-0

35. Ortiz MV, Gerdemann U, Raju SG, Henry D, Smith S, Rothenberg SM, et al. Activity of the Highly Specific RET Inhibitor Selpercatinib (LOXO-292) in Pediatric Patients With Tumors Harboring RET Gene Alterations. JCO Precis Oncol (2020) 4:PO.19.00401. doi: 10.1200/PO.19.00401

36. Solomon BJ, Tan L, Lin JJ, Wong SQ, Hollizeck S, Ebata K, et al. RET Solvent Front Mutations Mediate Acquired Resistance to Selective RET Inhibition in RET-Driven Malignancies. J Thorac Oncol (2020) 15:541-9. doi: 10.1016/ j.jtho. 2020.01 .006

37. Manfredi GI, Dicitore A, Gaudenzi G, Caraglia M, Persani L, Vitale G. PI3K/ Akt/mTOR Signaling in Medullary Thyroid Cancer: A Promising Molecular Target for Cancer Therapy. Endocrine (2015) 48:363-70. doi: 10.1007/s12020014-0380-1

38. Rapa I, Saggiorato E, Giachino D, Palestini N, Orlandi F, Papotti M, et al. Mammalian Target of Rapamycin Pathway Activation is Associated to RET Mutation Status in Medullary Thyroid Carcinoma. J Clin Endocrinol Metab (2011) 96:2146-53. doi: 10.1210/jc.2010-2655

39. Lim SM, Chang H, Yoon MJ, Hong YK, Kim H, Chung WY, et al. A Multicenter, Phase II Trial of Everolimus in Locally Advanced or Metastatic Thyroid Cancer of All Histologic Subtypes. Ann Oncol (2013) 24:3089-94. doi: 10.1093/annonc/mdt379

40. Hanna GJ, Busaidy NL, Chau NG, Wirth LJ, Barletta JA, Calles A, et al. Genomic Correlates of Response to Everolimus in Aggressive RadioiodineRefractory Thyroid Cancer: A Phase II Study. Clin Cancer Res (2018) 24:154653. doi: 10.1158/1078-0432.CCR-17-2297

41. Do K, Speranza G, Bishop R, Khin S, Rubinstein L, Kinders RJ, et al. Biomarker-Driven Phase 2 Study of MK-2206 and Selumetinib (AZD6244, ARRY-142886) in Patients With Colorectal Cancer. Invest New Drugs (2015) 33:720-8. doi: 10.1007/s10637-015-0212-z

42. Agrawal N, Jiao YC, Sausen M, Leary R, Bettegowda C, Roberts NJ, et al. Exomic Sequencing of Medullary Thyroid Cancer Reveals Dominant and Mutually Exclusive Oncogenic Mutations in RET and RAS. J Clin Endocr Metab (2013) 98:E364-9. doi: 10.1210/jc.2012-2703

43. Barletta JA, Nose V, Sadow PM. Genomics and Epigenomics of Medullary Thyroid Carcinoma: From Sporadic Disease to Familial Manifestations. Endocr Pathol (2021) 32(1):35-43. doi: 10.1007/s12022-021-09664-3

44. Ciampi R, Romei C, Ramone T, Prete A, Tacito A, Cappagli V, et al. Genetic Landscape of Somatic Mutations in a Large Cohort of Sporadic Medullary Thyroid Carcinomas Studied by Next-Generation Targeted Sequencing. Iscience (2019) 20:324-+. doi: 10.1016/j.isci.2019.09.030

45. Moura MM, Cavaco BM, Leite V. RAS Proto-Oncogene in Medullary Thyroid Carcinoma. Endocr Relat Cancer (2015) 22:R235-52. doi: 10.1530/ERC-15-0070

46. Vaseva AV, Yohe ME. Targeting RAS in Pediatric Cancer: Is it Becoming a Reality? Curr Opin Pediatr (2020) 32:48-56. doi: 10.1097/MOP.0000000000000856

47. Singh H, Longo DL, Chabner BA. Improving Prospects for Targeting RAS. J Clin Oncol (2015) 33:3650-9. doi: 10.1200/JCO.2015.62.1052

48. Prior IA, Hood FE, Hartley JL. The Frequency of Ras Mutations in Cancer. Cancer Res (2020) 80:2969-74. doi: 10.1158/0008-5472.CAN-19-3682
49. Boichard A, Croux L, Al Ghuzlan A, Broutin S, Dupuy C, Leboulleux S, et al. Somatic RAS Mutations Occur in a Large Proportion of Sporadic RET-Negative Medullary Thyroid Carcinomas and Extend to a Previously Unidentified Exon. J Clin Endocrinol Metab (2012) 97:E2031-5. doi: 10.1210/jc.2012-2092

50. Elisei R, Cosci B, Romei C, Bottici V, Renzini G, Molinaro E, et al. Prognostic Significance of Somatic RET Oncogene Mutations in Sporadic Medullary Thyroid Cancer: A 10-Year Follow-Up Study. J Clin Endocrinol Metab (2008) 93:682-7. doi: 10.1210/jc.2007-1714

51. Hobbs GA, Der CJ, Rossman KL. RAS Isoforms and Mutations in Cancer at a Glance. J Cell Sci (2016) 129:1287-92. doi: 10.1242/jcs.182873

52. O’Bryan JP. Pharmacological Targeting of RAS: Recent Success With Direct Inhibitors. Pharmacol Res (2019) 139:503-11. doi: 10.1016/j.phrs.2018.10.021

53. Hong DS, Cabanillas ME, Wheler J, Naing A, Tsimberidou AM, Ye L, et al. Inhibition of the Ras/Raf/MEK/ERK and RET Kinase Pathways With the Combination of the Multikinase Inhibitor Sorafenib and the Farnesyltransferase Inhibitor Tipifarnib in Medullary and Differentiated Thyroid Malignancies. J Clin Endocrinol Metab (2011) 96:997-1005. doi: 10.1210/jc.2010-1899

54. Sherman SI, Clary DO, Elisei R, Schlumberger MJ, Cohen EE, Schoffski P, et al. Correlative Analyses of RET and RAS Mutations in a Phase 3 Trial of Cabozantinib in Patients With Progressive, Metastatic Medullary Thyroid Cancer. Cancer (2016) 122:3856-64. doi: 10.1002/cncr.30252

55. Moore AR, Rosenberg SC, McCormick F, Malek S. RAS-Targeted Therapies: Is the Undruggable Drugged? Nat Rev Drug Discovery (2020) 19:533-52. doi: 10.1038/s41573-020-0068-6

56. Canon J, Rex K, Saiki AY, Mohr C, Cooke K, Bagal D, et al. The Clinical KRAS (G12C) Inhibitor AMG 510 Drives Anti-Tumour Immunity. Nature (2019) 575:217-23. doi: 10.1038/s41586-019-1694-1

57. Hallin J, Engstrom LD, Hargis L, Calinisan A, Aranda R, Briere DM, et al. The KRAS(G12C) Inhibitor MRTX849 Provides Insight Toward Therapeutic Susceptibility of KRAS-Mutant Cancers in Mouse Models and Patients. Cancer Discovery (2020) 10:54-71. doi: 10.1158/2159-8290.CD-19-1167

58. Molina-Arcas M, Samani A, Downward J. Drugging the Undruggable: Advances on RAS Targeting in Cancer. Genes (Basel) (2021) 12(6):899. doi: 10.3390/genes 12060899

59. Hong DS, Fakih MG, Strickler JH, Desai J, Durm GA, Shapiro GI, et al. KRAS (G12C) Inhibition With Sotorasib in Advanced Solid Tumors. N Engl J Med (2020) 383:1207-17. doi: 10.1056/NEJMoa1917239

60. Tran E, Robbins PF, Lu YC, Prickett TD, Gartner JJ, Jia L, et al. T-Cell Transfer Therapy Targeting Mutant KRAS in Cancer. N Engl J Med (2016) 375:2255-62. doi: 10.1056/NEJMoa1609279

61. Wang QJ, Yu Z, Griffith K, Hanada K, Restifo NP, Yang JC. Identification of T-Cell Receptors Targeting KRAS-Mutated Human Tumors. Cancer Immunol Res (2016) 4:204-14. doi: 10.1158/2326-6066.CIR-15-0188

62. Veatch JR, Jesernig BL, Kargl J, Fitzgibbon M, Lee SM, Baik C, et al. Endogenous CD4(+) T Cells Recognize Neoantigens in Lung Cancer Patients, Including Recurrent Oncogenic KRAS and ERBB2 (Her2) Driver Mutations. Cancer Immunol Res (2019) 7:910-22. doi: 10.1158/2326-6066.CIR-18-0402

63. Sim MJW, Lu J, Spencer M, Hopkins F, Tran E, Rosenberg SA, et al. HighAffinity Oligoclonal TCRs Define Effective Adoptive T Cell Therapy Targeting Mutant KRAS-G12D. Proc Natl Acad Sci USA (2020) 117:12826-35. doi: 10.1073/pnas.1921964117

64. Papotti M, Kumar U, Volante M, Pecchioni C, Patel YC. Immunohistochemical Detection of Somatostatin Receptor Types 1-5 in Medullary Carcinoma of the Thyroid. Clin Endocrinol (Oxf) (2001) 54:641-9. doi: 10.1046/j.1365-2265.2001.01175.x

65. Treglia G, Tamburello A, Giovanella L. Detection Rate of Somatostatin Receptor PET in Patients With Recurrent Medullary Thyroid Carcinoma: A Systematic Review and a Meta-Analysis. Hormones (Athens) (2017) 16:36272. doi: 10.14310/horm.2002.1756

66. Iten F, Muller B, Schindler C, Rochlitz C, Oertli D, Macke HR, et al. Response to [90Yttrium-DOTA]-TOC Treatment is Associated With Long-Term Survival Benefit in Metastasized Medullary Thyroid Cancer: A Phase II Clinical Trial. Clin Cancer Res (2007) 13:6696-702. doi: 10.1158/1078-0432.CCR-07-0935

67. Parghane RV, Naik C, Talole S, Desmukh A, Chaukar D, Banerjee S, et al. Clinical Utility of (177) Lu-DOTATATE PRRT in Somatostatin ReceptorPositive Metastatic Medullary Carcinoma of Thyroid Patients With Assessment of Efficacy, Survival Analysis, Prognostic Variables, and Toxicity. Head Neck (2020) 42:401-16. doi: 10.1002/hed.26024 
68. Maghsoomi Z, Emami Z, Malboosbaf R, Malek M, Khamseh ME. Efficacy and Safety of Peptide Receptor Radionuclide Therapy in Advanced RadioiodineRefractory Differentiated Thyroid Cancer and Metastatic Medullary Thyroid Cancer: A Systematic Review. BMC Cancer (2021) 21:579. doi: 10.1186/ s12885-021-08257-x

69. Grossrubatscher E, Fanciulli G, Pes L, Sesti F, Dolci C, de Cicco F, et al. Advances in the Management of Medullary Thyroid Carcinoma: Focus on Peptide Receptor Radionuclide Therapy. J Clin Med (2020) 9(11):3507. doi: $10.3390 /$ jcm 9113507

70. Schott M, Seissler J, Lettmann M, Fouxon V, Scherbaum WA, Feldkamp J. Immunotherapy for Medullary Thyroid Carcinoma by Dendritic Cell Vaccination. J Clin Endocrinol Metab (2001) 86:4965-9. doi: 10.1210/jcem.86.10.7949

71. Bilusic M, Heery CR, Arlen PM, Rauckhorst M, Apelian D, Tsang KY, et al. Phase I Trial of a Recombinant Yeast-CEA Vaccine (GI-6207) in Adults With Metastatic CEA-Expressing Carcinoma. Cancer Immunol Immunother (2014) 63:225-34. doi: 10.1007/s00262-013-1505-8

72. Del Rivero J, Donahue RN, Marte JL, Gramza AW, Bilusic M, Rauckhorst M, et al. A Case Report of Sequential Use of a Yeast-CEA Therapeutic Cancer Vaccine and Anti-PD-L1 Inhibitor in Metastatic Medullary Thyroid Cancer. Front Endocrinol (Lausanne) (2020) 11:490. doi: 10.3389/fendo.2020.00490

73. Bhoj VG, Li L, Parvathaneni K, Zhang Z, Kacir S, Arhontoulis D, et al. Adoptive T Cell Immunotherapy for Medullary Thyroid Carcinoma Targeting
GDNF Family Receptor Alpha 4. Mol Ther Oncolytics (2021) 20:387-98. doi: 10.1016/j.omto.2021.01.012

Conflict of Interest: The authors declare that the research was conducted in the absence of any commercial or financial relationships that could be construed as a potential conflict of interest.

The reviewer MAZ declared a shared affiliation, though no other collaboration, with the authors to the handling Editor.

Publisher's Note: All claims expressed in this article are solely those of the authors and do not necessarily represent those of their affiliated organizations, or those of the publisher, the editors and the reviewers. Any product that may be evaluated in this article, or claim that may be made by its manufacturer, is not guaranteed or endorsed by the publisher.

Copyright (C) 2021 Okafor, Hogan, Raygada, Thomas, Akshintala, Glod and Del Rivero. This is an open-access article distributed under the terms of the Creative Commons Attribution License (CC BY). The use, distribution or reproduction in other forums is permitted, provided the original author(s) and the copyright owner(s) are credited and that the original publication in this journal is cited, in accordance with accepted academic practice. No use, distribution or reproduction is permitted which does not comply with these terms. 